\title{
Investigation of the thermal conductivities across metal-insulator transition in polycrystalline $\mathrm{VO}_{2}$
}

\author{
CHEN JiKun ${ }^{1,2}$, LIU XinLing ${ }^{2}$, YUAN Xun ${ }^{2}$, ZHANG YuLiang ${ }^{1}$, GAO YanFeng ${ }^{2}$, \\ ZHOU YanFei ${ }^{2}$, LIU RuiHeng ${ }^{2}$, CHEN LiDong ${ }^{2 *} \&$ CHEN NuoFu ${ }^{1 *}$ \\ ${ }^{1}$ School of Renewable Energy, North China Electric Power University, Beijing 102206, China; \\ ${ }^{2}$ CAS Key laboratory of Materials for Energy Conversion, Shanghai Institute of Ceramics, Chinese Academy of Sciences, \\ Shanghai 200050, China
}

Received December 15, 2011; accepted February 29, 2012; published online July 2, 2012

\begin{abstract}
Previous reports about the thermal conductivities of $\mathrm{VO}_{2}$ showed various temperature dependences across metal-insulator transition (MIT) temperature. In this work, polycrystalline $\mathrm{VO}_{2}$ samples were fabricated by spark plasma sintering of $\mathrm{VO}_{2}$ powder. Temperature dependences of their thermal conductivities were investigated using laser flash technique, and the thermal conductivity showed a significant decrease trend from metal-phase to insulator phase. Electrical transport properties were investigated to confirm both carrier and lattice contribution to the thermal conductivity. It is found that the lattice thermal conductivity decreased significantly across MIT point, which may be caused by soft phonon mode in metal phase of $\mathrm{VO}_{2}$.
\end{abstract}

thermal conductivity, phase transformation, $\mathrm{VO}_{2}$

Citation: Chen J K, Liu X L, Yuan X, et al. Investigation of the thermal conductivities across metal-insulator transition in polycrystalline VO $\mathrm{O}_{2}$. Chin Sci Bull, 2012, 57: 3393-3396, doi: 10.1007/s11434-012-5294-9

The metal-insulator transition (MIT) in vanadium dioxide $\left(\mathrm{VO}_{2}\right)$ has received considerable attentions due to its potential applications in optical waveguides and high-speed electronics [1,2]. At the MIT temperature around $68^{\circ} \mathrm{C}, \mathrm{VO}_{2}$ exhibits a phase transition from high-temperature metallic behavior to low-temperature insulating or semi-conductive behavior [3]. Along with this transition, the optical, magnetic and electrical transport properties of vanadium dioxide change dramatically within a narrow temperature interval, which have received intensive investigation in the last decade [4-6]. However, the thermophysical properties of $\mathrm{VO}_{2}$, such as thermal conductivity, near the MIT temperature have been less concerned. As to further satisfy the development of $\mathrm{VO}_{2}$ based high speed energy switch devices, investigation and comprehension of the thermal transportation properties of $\mathrm{VO}_{2}$ are still needed [7].

Previous reports about the thermal conductivity of $\mathrm{VO}_{2}$

*Corresponding authors (email: nfchen@ncepu.edu.cn; chenlidong@mail.sic.ac.cn) showed various values and temperature dependences across MIT temperature [8-14]. Berglund et al. [8] reported that the thermal conductivity of bulk $\mathrm{VO}_{2}$ sample changed little when passing through the transition point, whereas Andreev et al. [9] reported a marked decrease from insulating phase to metallic phase. Recently, Oh et al. [10] applied a novel method to the thermal conductivity measurement of $\mathrm{VO}_{2}$ thin films and found that the thermal conductivity would increase by $60 \%$ in the metallic phase, which could be comprehended as the contribution of the increased electrical conductivity assuming that the lattice contribution of thermal conductivity remains the same before and after the MIT point. The variety of these results makes it necessary to investigate more about the thermal conductivity of $\mathrm{VO}_{2}$. In this work, polycrystalline $\mathrm{VO}_{2}$ samples were fabricated by spark plasma sintering (SPS) using micro-scaled $\mathrm{VO}_{2}$ powder. The thermal conductivity of as-produced $\mathrm{VO}_{2}$ sample near the MIT temperature was measured using laser flash method, and the temperature dependence of lattice thermal 
conductivity was also investigated.

\section{Experimental}

$\mathrm{VO}_{2}$ powder used for SPS was fabricated using the method reported by Peng et al. [11]. The obtained $\mathrm{VO}_{2}$ powder was sintered into a dense polycrystalline solid using the spark plasma sintering technique (SPS-2040) at $900^{\circ} \mathrm{C}$ under 20 $\mathrm{MPa}$ for $10 \mathrm{~min}$ in a graphite die. Relatively high-density samples ( $>98 \%$ of the theoretical density) with diameter of $10 \mathrm{~mm}$ and thickness of $1.5 \mathrm{~mm}$ were obtained in the present study. X-ray diffraction data was collected on a diffractometer (Rigaku, RINT2000) with $\mathrm{CuK} \alpha$ radiation at room temperature to examine the phase purity and structure. The measurement of thermal diffusivity $(\lambda)$ was carried out using the laser flash method in a flowing $\mathrm{Ar}$ atmosphere (Netzsch LFA 427), and the measurement was performed in the temperature range of $20-150^{\circ} \mathrm{C}$. The sample of $\mathrm{VO}_{2}$ was sprayed with graphite in each surface when carrying out the thermal diffusivity measurement. The heat capacity $C_{\mathrm{p}}$ of the sample after SPS treatment was measured by differential scanning calorimetry (PE DSC-2C) in the temperature range of $25-150^{\circ} \mathrm{C}$. Thermal conductivity $(\kappa)$ was calculated from the relationship $\kappa=\rho \lambda C_{\mathrm{p}}$, where $\lambda$ is the thermal diffusivity coefficient, $C_{\mathrm{p}}$ is the heat capacity and $\rho$ is the density of the material. Samples used for electrical transport measurements were cut into rectangular bars with the approximate dimensions of $1 \mathrm{~mm} \times 2 \mathrm{~mm} \times 10 \mathrm{~mm}$, and the measurement of electrical conductivity and Seebeck coefficient were carried out using ZEM-3 (ULVAC-RLKO) under the temperature range of $20-150^{\circ} \mathrm{C}$.

\section{Results and discussion}

Figures 1 and 2 show the SEM image of fractured surface

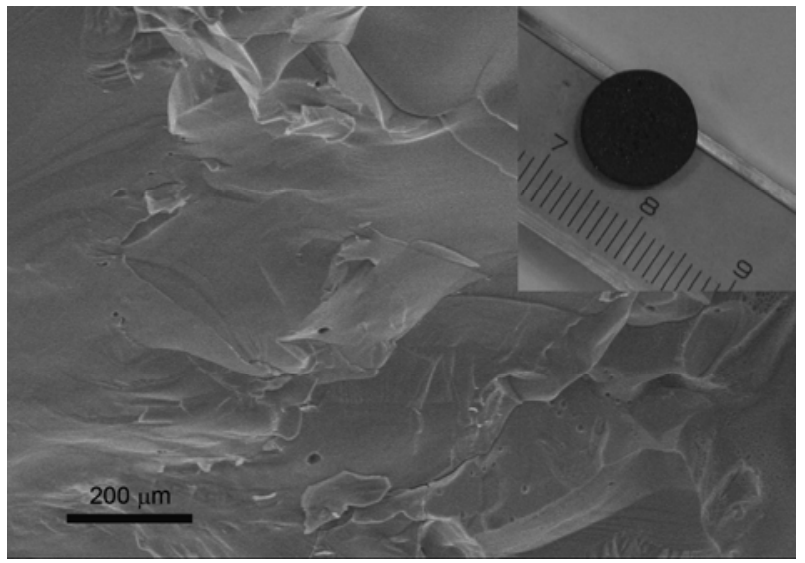

Figure 1 SEM image of the fracture surface morphology of the $\mathrm{VO}_{2}$ sample after SPS, with inset showing the photo of as-SPSed $\mathrm{VO}_{2}$ sample.

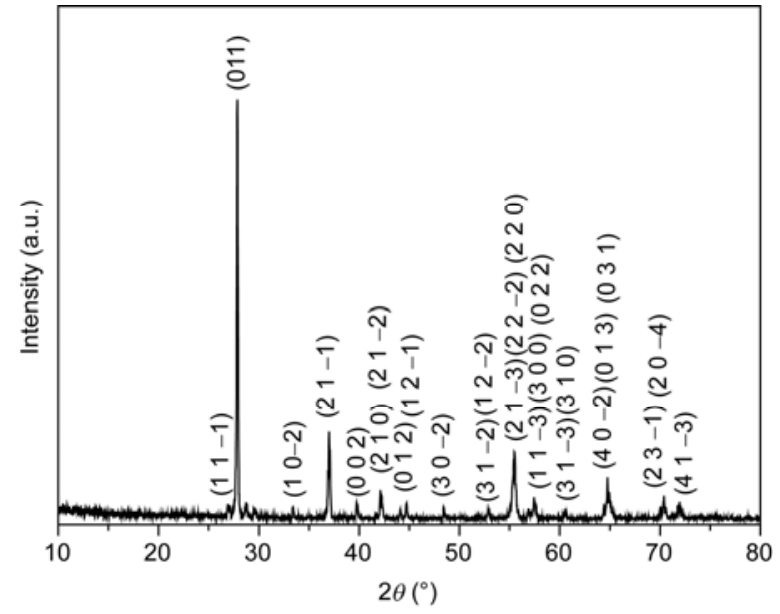

Figure 2 XRD pattern of as-SPSed $\mathrm{VO}_{2}$ (measured at room temperature), with main peaks indexed as monoclinic phase.

and the XRD pattern of the $\mathrm{VO}_{2}$ sample after SPS, respectively. During the SPS process, the pulsed DC current directly passes through the graphite die and the powder compact, which generates the heat internally and enhances the densification of the sample. Besides, the decreased sintering time in SPS process maintains the origin chemical state of $\mathrm{VO}_{2}$ that is sensitive to the atmosphere in high temperature. It can be seen that the as-produced $\mathrm{VO}_{2}$ sample possesses polycrystalline structure with monoclinic structure, which accords with the low-temperature phase of $\mathrm{VO}_{2}$. The grain size of the sample was around tens of micrometers, and the density of the as-sintered sample reached $4.62 \mathrm{~g} \mathrm{~cm}^{-3}$, compared with the theoretical density of $4.65 \mathrm{~g} \mathrm{~cm}^{-3}$.

Thermal conductivity of as-produced $\mathrm{VO}_{2}$ sample is shown in Figure 3, together with those reported previously. In this work, thermal conductivity of the polycrystalline $\mathrm{VO}_{2}$ sample is about $3.5 \mathrm{~W} \mathrm{~m}^{-1} \mathrm{~K}^{-1}$ in the insulating phase, which stood at a similar magnitude with the recent result obtained by $\mathrm{Oh}$ et al. [10] in $\mathrm{VO}_{2}$ thin films. As for higher

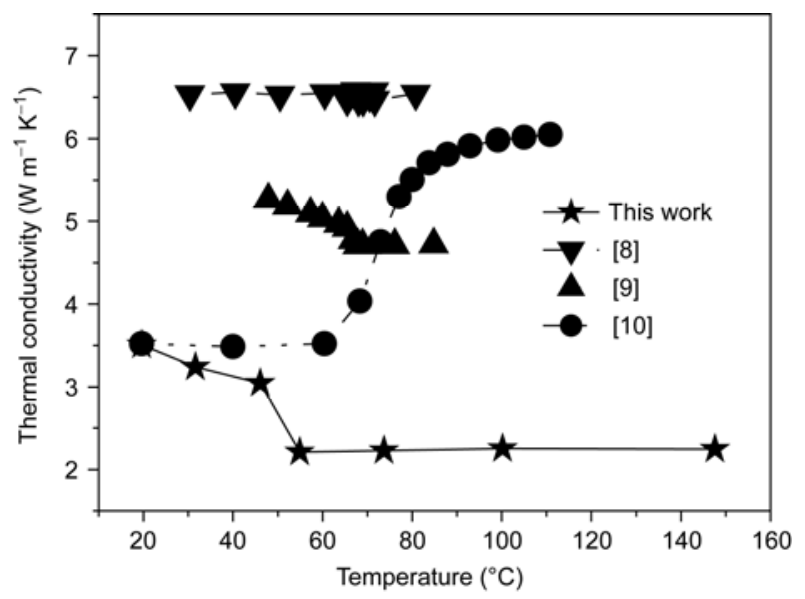

Figure 3 Thermal conductivity of the as-sintered $\mathrm{VO}_{2}$ sample, compared with results from previous reports. 
temperature metallic phase, the thermal conductivity showed a significant decrease about $35 \%$ that accords with the report from Andreev [9]. The thermal conductivity was measured during a heating process. To testify the reversibility of this change, the thermal conductivity was measured again when temperature dropped to around $20^{\circ} \mathrm{C}$, and the thermal conductivity returned to the same magnitude of the low temperature phase.

The as-measured thermal conductivity $\kappa$ is a sum of two contributions: lattice part $\kappa_{\mathrm{L}}$ and carrier part $\kappa_{\mathrm{c}} . \kappa_{\mathrm{c}}$ can be estimated by the Wiedemann-Franz law as $\kappa_{\mathrm{c}}=L_{0} \sigma T$, where $L_{0}$ is the Lorenz number $\left(2.45 \times 10^{-8} \mathrm{~V}^{2} \mathrm{~K}^{-2}\right)$. That the temperature dependence of thermal conductivity across the MIT point is different in this work compared with that in Oh's should be attributed to the different carrier contribution of thermal conductivity. In order to further calculate the lattice contribution to thermal conductivity, temperature dependence of the electrical transport properties of the samples was also measured. As shown in Figure 4, the negative Seebeck coefficient through the whole temperature range indicates that the main carrier is electron in both phases. Around $65^{\circ} \mathrm{C}$, the electrical conductivity of the sample increased about one magnitude within a narrow temperature range, while the Seebeck coefficient decreased significantly, which indicates that the carrier concentration increased significantly by the phase transition from insulating phase to metallic phase. However, the enhancement of the electrical

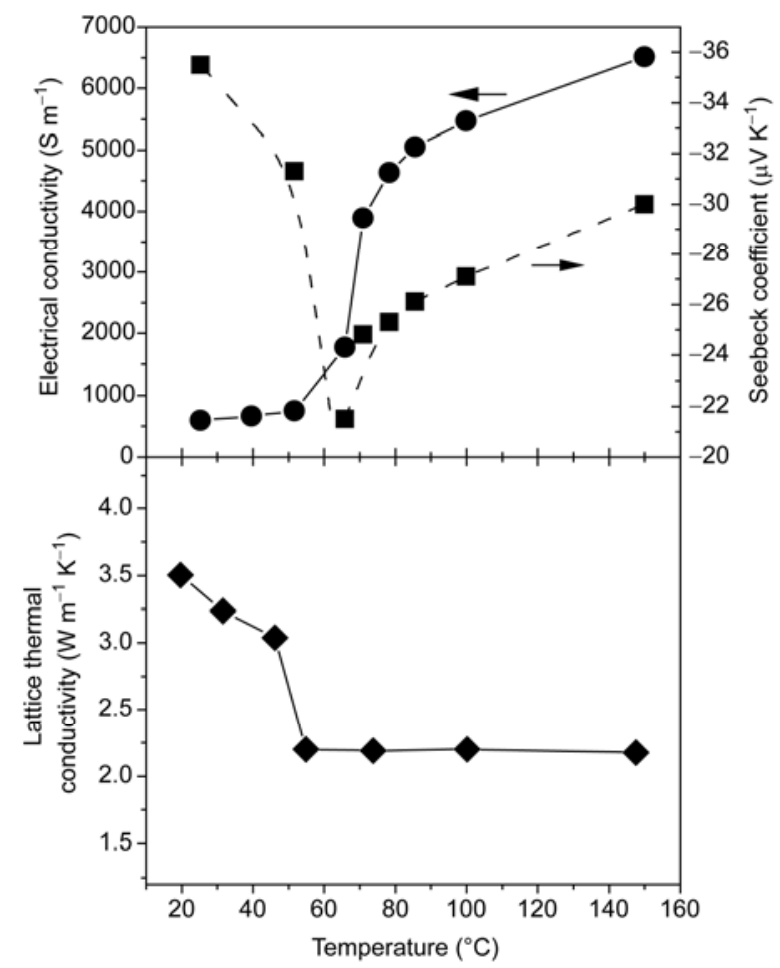

Figure 4 Electrical conductivity, Seebeck coefficient and lattice thermal conductivity of the $\mathrm{VO}_{2}$ sample in the temperature range of $20-150^{\circ} \mathrm{C}$. The solid line with round point shows the electrical conductivity, while the dash line with square point shows the Seebeck coefficient. conductivity in our sample is not as large as what were reported in single crystalline or thin film $\mathrm{VO}_{2}$ samples. The metallic phase of the $\mathrm{VO}_{2}$ sample showed semiconducting character, since the electrical conductivity was found to increase with temperature in the present sample. This indicates that there may be localized states or electrons, which could be caused by defects like grain boundaries and pores. By heat excitation, these localized electrons would become carriers and contribute to the electrical conductivity. What also needs to mention is that the phase transition temperature shown in thermal conductivity measurement was prior to that measured in the electrical conductivity. This is mainly caused by the used laser flashing, which would generate extra heat to cause a temperature rise of $2-5^{\circ} \mathrm{C}$ of the sample compared with the background temperature, and will induce the phase transition when background temperature was close to the MIT point.

The calculated lattice thermal conductivity of as-produced $\mathrm{VO}_{2}$ sample is shown in the downside picture in Figure 4. The lower lattice thermal conductivity $\kappa_{\mathrm{L}}$ in the metallic phase of $\mathrm{VO}_{2}$ was possibly caused by the soft phonon mode in the high temperature phase, which has been proved directly in the XRD scatting experiment [12]. On the other hand, the high temperature phase of $\mathrm{VO}_{2}$ has lower acoustic velocity according to dispersion relationship, which has been observed by measuring Rayleigh velocity before and after phase transition $[13,14]$. The significant decrease of lattice thermal conductivity $\kappa_{\mathrm{L}}$ counts for the negative temperature dependence of the total thermal conductivity across the MIT point, since the carrier contribution $\kappa_{\mathrm{c}}$ is low in our samples.

\section{Conclusion}

In summary, polycrystalline $\mathrm{VO}_{2}$ samples with high relative density were fabricated by spark plasma sintering (SPS) using micrometer-sized $\mathrm{VO}_{2}$ powder. The temperature dependence of the thermal conductivity across MIT point was investigated using standard laser-flash method. For our samples, the total thermal conductivity showed $30 \%$ decrease from low-temperature insulating phase to high temperature metallic phase. This negative temperature dependence of thermal conductivity across MIT point is caused by the decrease of lattice thermal conductivity across the phase transition, since the carrier contribution of thermal conductivity is low. The different variation trends of thermal conductivity of $\mathrm{VO}_{2}$ across MIT point in previous reports might be caused by the difference in carrier contribution to the thermal conductivity, which is sensitive to the structure and defects of the samples.

This work was supported by the National Natural Science Foundation of China (50821004 and 61076051) and the Program of Shanghai Subject 
Chief Scientist (09XD1404400). Assistance by Prof. Zhang Xiulan of Institute of Semiconductors, Chinese Academy of Sciences are acknowledged.

1 Lee M J, Park Y. Two series oxide resistors applicable to high speed and high density nonvolatile memory. Adv Mater, 2007, 19: 3919-3923

2 Rini M, Hao Z. Optical switching in $\mathrm{VO}_{2}$ films by below-gap excitation. Appl Phys Lett, 2008, 92: 181904

3 Morin J. Oxides which show a metal-to-insulator transition at the Neel temperature. Phys Rev Lett, 1959, 3: 34-36

4 Donev E U, Lopez R. Confocal Raman microscopy across the metalinsulator transition of single vanadium dioxide nanoparticles. Nano Lett, 2009, 9: 702-706

5 Qazilbash M M, Brehm M. Infrared spectroscopy and nano-imaging of the insulator-to-metal transition in vanadium dioxide. Phys Rev B, 2009, 79: 075107

6 Lee M H. Thermochromic glazing of windows with better luminous solar transmittance. Sol Energy Mater Sol Cells, 2002, 71: 537-540

7 Chang C W, Okawa D. Solid-state thermal rectifier. Science, 2006,
314: 1121-1124

8 Berglund $\mathrm{C} \mathrm{N}$, Guggenheim $\mathrm{H} \mathrm{J}$. Electronic properties of $\mathrm{VO}_{2}$ near semiconductor-metal transition. Phys Rev B, 1969, 185: 1022-1033

9 Andreev V N, Chudnovskii $\mathrm{F}$ A. Thermal conductivity of $\mathrm{VO}_{2}, \mathrm{~V}_{3} \mathrm{O}_{5}$, and $\mathrm{V}_{2} \mathrm{O}_{3}$. Phys Status Solidi (a), 1978, 48: K153-K156

10 Oh D W, Ko C Y. Thermal conductivity and dynamic heat capacity across the metal-insulator transition in thin film $\mathrm{VO}_{2}$. Appl Phys Lett, 2010, 96: 151906

11 Peng Z F, Jiang W. Synthesis and electrical properties of tungstendoped vanadium dioxide nanopowders by thermolysis. J Phys Chem C, 2007, 111: 1119-1122

12 Terauchi H, Cohen J B. Diffuse X-ray scattering due to lattice instability near matal-semiconductor transition in $\mathrm{VO}_{2}$. Phys Rev B, 1978, 17: 2494-2496

13 Maurer D, Leue A. Elastic behavior near the metal-insulator transition of $\mathrm{VO}_{2}$. Phys Rev B, 1999, 60: 13249-13252

14 Chen $\mathrm{J}$ K, Liu X L. Deoxidization of $\mathrm{V}_{2} \mathrm{O}_{5}$ powder into $\mathrm{VO}_{2}$ assisted by an electrochemical lithium intercalation technique. Int $\mathrm{J}$ Appl Ceram Technol, 2011, doi: 10.1111/j.1744-7402.2011.00674.x

Open Access This article is distributed under the terms of the Creative Commons Attribution License which permits any use, distribution, and reproduction in any medium, provided the original author(s) and source are credited. 